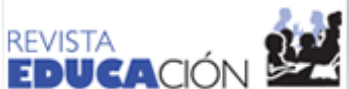

Revista Educación

ISSN: 0379-7082

ISSN: 2215-2644

revedu@gmail.com

Universidad de Costa Rica

Costa Rica

\section{Influencia de la educación emprendedora sobre la intención de emprender del alumnado universitario}

\author{
Araya-Pizarro, Sebastián Cristóbal \\ Influencia de la educación emprendedora sobre la intención de emprender del alumnado universitario \\ Revista Educación, vol. 45, núm. 2, 2021 \\ Universidad de Costa Rica, Costa Rica \\ Disponible en: https://www.redalyc.org/articulo.oa?id=44066178029 \\ DOl: https://doi.org/10.15517/revedu.v45i1.43748
}

\section{(c) (1) 90}

Esta obra está bajo una Licencia Creative Commons Atribución-NoComercial-SinDerivar 3.0 Internacional. 
Revisiones bibliográficas

\title{
Influencia de la educación emprendedora sobre la intención de emprender del alumnado universitario
}

\author{
Impact of Entrepreneurship Education on University Students with Entrepreneurial Intentions \\ Sebastián Cristóbal Araya-Pizarro \\ Universidad de La Serena, Chile \\ saraya@userena.cl \\ DOI: https://doi.org/10.15517/revedu.v45i1.43748 \\ Redalyc: https://www.redalyc.org/articulo.oa? \\ $\mathrm{id}=44066178029$
}

(iD) https://orcid.org/0000-0002-5857-8441

Recepción: 20 Septiembre 2020

Aprobación: 25 Noviembre 2020

\section{Resumen:}

Dada la importancia de la enseñanza para impulsar el emprendimiento, el presente estudio planteó por objetivo analizar la influencia de la educación emprendedora sobre la intención de emprender del estudiantado universitario. Se realizó una revisión de la literatura (periodo 2010-2020) mediante una ecuación de búsqueda en la base de datos Web of Science, que permitió identificar 80 publicaciones relevantes. La información seleccionada fue revisada a través de la técnica de análisis documental, que incluyó el análisis de contenidos, el examen de indicadores bibliométricos y de redes de coocurrencia de palabras claves. Los hallazgos revelan que la mayoría de los estudios teórico-empíricos aplican el Modelo del Comportamiento Planificado de Ajzen, con el cual se indaga la influencia de diversos predictores individuales y contextuales sobre la intención de emprender. Además, destaca la utilización de un paradigma de investigación positivista, basado en un enfoque cuantitativo y de corte transversal, que aplica como técnicas de estudio las encuestas y el análisis estadístico multivariante (ecuaciones estructurales). Por lo demás, los resultados muestran que los trabajos provienen principalmente de Asia y Europa; se encuentran pocos artículos de países latinoamericanos. En relación con la influencia de la educación emprendedora sobre la intención de emprender, se observa un efecto significativo y positivo, pero dependiente del contexto de estudio y del tipo de enseñanza impartida. Por tanto, se sostiene que los programas de emprendimiento efectivos se vincularían a una formación emprendedora más dinámica, práctica y alineada al contexto y características del cuerpo estudiantil. Finalmente, se recomienda profundizar el escrutinio de aquellos factores pedagógicos que influyen en la actitud del estudiantado hacia el emprendimiento.

Palabras ClaVe: Educación emprendedora, Intención emprendedora, Análisis bibliométrico, Estudiantado universitario.

\section{Abstract:}

Given the importance of fostering entrepreneurship, this study analyzes the influence of entrepreneurial education on university students with an entrepreneurial penchant. A literature review (2010-2020 period) using a search equation in the Web of Science database, identified 80 relevant publications, subsequently, reviewed using a documentary analysis technique that included content analysis, examination of bibliometric indicators and keyword co-occurrences. Findings reveal that most of the theoreticalempirical studies used the Ajzen's Planned Behavior Model to identify individual and contextual predictors to determine entrepreneurial intention. It also highlights the use of a positivist research paradigm based on a quantitative and cross-sectional approach using surveys and multivariate statistical analysis (structural equations) as study methodologies. It was determined that most studies stem from Asia and Europe with few originating in Latin America. A significant and positive effect was observed with regards to the impact of entrepreneurial education on entrepreneurship intention. However, this was dependent on the context of the study and the type of training provided. Effective entrepreneurship programs were linked to more dynamic and practical entrepreneurial training, aligned to the context and characteristics of the student body. We encourage a more profound analysis of pedagogical factors that, ultimately, influence student attitudes towards entrepreneurship.

KEYWORDS: Entrepreneurship Education, Entrepreneurial Intention, Bibliometric Analysis, University Students.

\section{INTRODUCCIÓN}

El emprendimiento es reconocido como un atributo clave para el crecimiento económico de las naciones, por lo que el estudio de los factores determinantes de su desarrollo se ha vuelto esencial. La educación 
emprendedora (EE), por su parte, se identifica como el medio para impulsar la actividad empresarial y el desarrollo socioeconómico de los territorios (Soria-Barreto, Zuniga-Jara y Ruiz-Campo, 2016; Stamboulis y Barlas, 2014; Jena, 2020); responsable de formar personas con competencias especiales, estimadas como imprescindibles para la adaptación en los nuevos mercados laborales. Es un factor clave para aumentar la intención emprendedora (IE) del estudiantado, es decir, de aquel estado mental que dirige la atención, experiencia y acción a emprender (Çera y Çera, 2020; Lanero, Vázquez, Gutiérrez y García, 2011; Paray y Kumar, 2020).

El vínculo entre la educación y el emprendimiento se ha estudiado desde diferentes perspectivas; se advierte que cuanto más intensiva es la enseñanza del emprendimiento en las instituciones de educación, más probabilidades existen de que el alumnado haga el esfuerzo por iniciar una empresa y, de esa forma, contribuir al desarrollo de la economía. No obstante, aunque algunos estudios han propuesto una relación positiva entre la educación y las actitudes, intenciones o acciones empresariales, la evidencia no es sólida y parece depender de las categorías de educación emprendedora y sus programas académicos (Čapienė y Ragauskaitė, 2017). Así, aun cuando la relación entre la educación y el emprendimiento ha sido tratada extensamente, todavía no existe un acuerdo respecto del efecto que provoca la enseñanza del emprendimiento sobre la IE del alumnado de educación superior (da Silva Moreira, Loiola y Guedes, 2017).

En este contexto, el presente artículo planteó por objetivo analizar la influencia de la EE sobre la IE del estudiantado universitario. El estudio contribuye a responder una de las preguntas clave en la investigación sobre el emprendimiento, la cual señala: ¿ por qué algunas personas emprenden y otras no? Es decir, corroborar la premisa particular de que el espíritu emprendedor puede ser potenciado por medio de la educación, especialmente, la educación emprendedora.

Por lo demás, se incluye un examen bibliométrico que abarcó diferentes indicadores de medición, tales como revistas y editoriales con máximo número de publicaciones y citaciones, evolución de publicaciones por año, artículos más citados, áreas de investigación, redes de coocurrencias de palabras clave, países y continentes más prolíferos en el estudio de la temática. Con ello se pretende generar un marco referencial básico sobre la teoría, metodología y efecto de la EE sobre la IE.

La bibliometría reviste, como indica Montilla (2012), un rol fundamental por presentar uno de los análisis métricos de datos más robustos del campo informacional, de lo que resulta un método universal y confiable para medir la productividad de un área de conocimiento específico y así obtener resultados fiables para la toma de decisiones.

\section{Metodología}

Para analizar la influencia de la enseñanza del emprendimiento sobre la intención emprendedora del alumnado universitario, se llevó a cabo una investigación de tipo descriptiva, basada en la revisión sistemática de la literatura (análisis documental). Para ello se seleccionó como herramienta de búsqueda la base de datos de Web of Science. De acuerdo con Norris y Oppenheim (2007), la selección de la base de datos para este tipo de estudios es un aspecto fundamental, porque permite garantizar la disponibilidad, pertinencia y confiabilidad de la información. Por tal razón, se eligió este repositorio, dado que es de gran visibilidad y en él se depositan publicaciones multidisciplinarias de alto impacto. Además, brinda opciones de búsqueda avanzada que permiten un escrutinio detallado de la información de los artículos consultados.

Los procedimientos de búsqueda en la base de datos se llevaron a cabo entre julio y agosto de 2020. Se identificaron 80 publicaciones relevantes respecto de la influencia de la EE sobre la IE en el alumnado universitario, derivadas de revistas especializadas en educación, emprendimiento y psicología, como, por ejemplo, Education y Training, Studies in Higher Education, Sustainability, Journal of Small Business Management, International Journal of Management Journal, Frontier in Psychology, entre otras. Se excluyeron 
los artículos teóricos o aquellos que informaron investigaciones con muestras que no son de estudiantes de universidades, como estudiantes de secundaria, representantes del empresariado o población general.

Los criterios de selección para los manuscritos fueron: 1) artículos de investigación de carácter científico publicados en revistas indexadas, lo que excluye la consulta de capítulos de libros, conferencias, enciclopedias, tesis, informes de patentes, noticias, entre otros; 2) artículos publicados durante los últimos diez años, es decir, el periodo comprendido entre los años 2010 y 2020 inclusive; y 3) publicaciones vinculadas directamente con las categorías de búsqueda: educación e intención emprendedora en educación superior universitaria.

Las palabras de búsqueda utilizadas (entrepreneurship education, entrepreneurial intention) para obtener los registros del periodo de estudio, es decir, desde 2010 hasta la actualidad, arrojaron un total inicial de más de 167 documentos. Luego de revisar cada uno de los resúmenes de las publicaciones y evaluar su pertinencia a la temática investigativa, se seleccionó un total de 80 artículos válidos. Específicamente, el algoritmo de búsqueda fue: entrep. educ. entrep. intent. univers*.

Luego de aplicada la ecuación, se procedió a realizar la clasificación de los datos arrojados, con el fin de analizar los indicadores de revisión sistemática. Cabe indicar que la información fue organizada en una base de datos compuesta de 44 campos de clasificación, entre los que destacan: título del artículo, autores, revista, editorial, palabras claves, resumen, metodología, influencia de la EE, citas, área de investigación, país, entre otras.

El examen bibliométrico incluyó: revistas más citadas y editoriales con máximo número de publicaciones, evolución de publicaciones por año, artículos más citados, áreas de investigación, países y continentes más prolíferos. Además, se realizó el estudio de las palabras clave, mediante el análisis de redes de coocurrencias. Los contenidos de los artículos, por su parte, fueron revisados en función de su opción teórica, metodológica e influencia de la EE sobre la IE.

Para el análisis de redes de coocurrencias de palabras clave, se utilizó el software VOS Viewer 1.6.9. Este programa es una herramienta de software gratuita que se utiliza para crear mapas basados en datos de red. Se puede utilizar para crear mapas de revistas o autores basados en los datos de cocitas o para crear mapas de palabras clave basados en los datos de coocurrencia (van Eck, Waltman, Dekker y van den Berg, 2010). Además, para el análisis estadístico de datos se usó el software IBM SPSS Statistics 24 y Tableau Public 2020.2.

\section{ANÁLISIS Y DISCUSIÓN DE RESULTADOS}

A continuación, se presentan los resultados del estudio divididos en dos secciones. Primero se exponen los indicadores derivados del análisis bibliométrico de la producción científica. Y, segundo, se muestran los hallazgos correspondientes al análisis del contenido de los artículos, según el escrutinio de los marco teóricos, metodológicos y resultados obtenidos.

\section{Revistas y editoriales con máximo número de publicaciones}

Los 80 artículos de este estudio pertenecen a 52 revistas. El 80\% de las citas se concentran en seis revistas, de ellas, las tres más importantes son: Journal of Small Business Management, con 330 citas; International Entrepreneurship and Management Journal, con 278 citas; y Studies in Higher Education, con 95 citas (Véase la Tabla 1). 
TABLA 1

Revistas con más citaciones sobre la influencia de la EE sobre la IE

\begin{tabular}{lll}
\hline Revista & $\begin{array}{l}\text { Total de } \\
\text { citaciones }\end{array}$ & Porcentaje \\
\hline Journal of Small Business Management & 330 & 30,7 \\
International Entrepreneurship and Management & 278 & 25,9 \\
Journal & 95 & 8,8 \\
Studies in Higher Education & 80 & 7,4 \\
Technological Forecasting and Social Change & 50 & 4,7 \\
Education and Training & 27 & 2,5 \\
Sustainability & 24 & 2,2 \\
International Journal of Engineering Education & 24 & 2,2 \\
Journal of Intellectual Capital & 17 & 1,6 \\
Formación Universitaria & 14 & 1,3 \\
Journal of Small Business and Enterprise & & 1,2 \\
Development & 13 & 1,1 \\
Educational Studies & 12 & \\
Rusc-Universities and Knowledge Society Journal & &
\end{tabular}

Fuente: Elaboración propia

Entre las revistas principales, doce pertenecen a las editoriales Emerald, seis a la editorial Routledge Journals, Taylor y Francis, y tres a Inderscience. El resto de revistas pertenecen a editoriales como Springer, Wiley, Elsiever, MDPI, entre otras. La revista más importante, Education and Training (Educ Train), que ha publicado seis artículos, pertenece a la editorial Emerald Group Publishing Ltd. La Figura 1 ilustra las quince principales revistas, que publicaron la mayor cantidad de artículos de la influencia de la educación sobre la IE del estudiantado universitario.

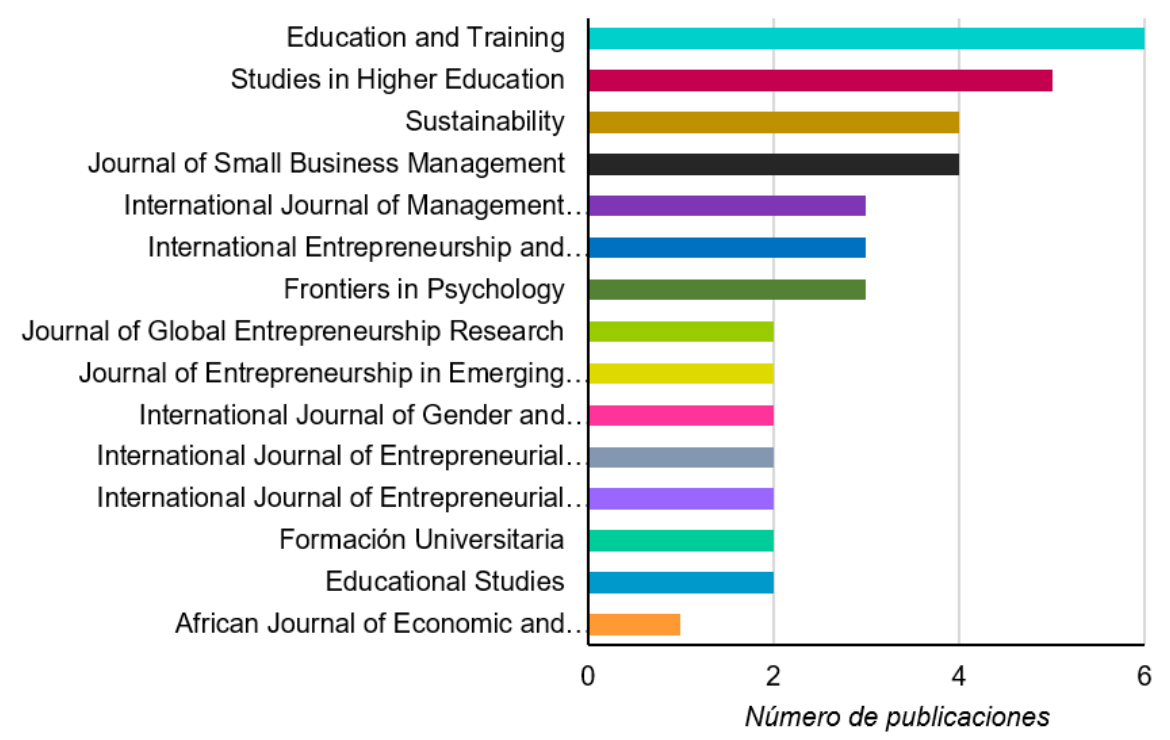

FIGURA 1

Revistas con mayor volumen de publicaciones en la materia Fuente: Elaboración propia

Por lo demás, las áreas de investigación principales fueron Economía y Negocios, y Educación e Investigación Educativa. Las cuales, en total, abarcan cerca del 74\% del total de las categorías de investigación (59) y el 84\% (905) del total de las citaciones (Véase la Tabla 2). 
TABLA 2

Total de revistas y citaciones según áreas de investigación

\begin{tabular}{lll}
\hline Influencia & $\mathrm{N}^{\circ}$ revistas & T.C. \\
\hline Agriculture & 1 & 4 \\
Area Studies & 1 & 1 \\
Business y Economics & 35 & 716 \\
Business y Economics; Education y Educational Research & 3 & 3 \\
Business y Economics; Public Administration & 1 & 80 \\
Education y Educational Research & 24 & 189 \\
Education y Educational Research; Engineering & 1 & 24 \\
Education y Educational Research; Environmental Sciences y & 1 & \\
Ecology & 2 & 4 \\
Engineering & 1 & 17 \\
Environmental Sciences y Ecology & 1 & 0 \\
Government y Law & 4 & 5 \\
Psychology & 1 & 4 \\
Science y Technology - Other Topics & 4 & 27 \\
Science y Technology and Other Topics & & \\
\hline
\end{tabular}

Fuente: Elaboración propia

Nota: T.C. $=$ Total de citaciones

Nota: T.C. $=$ Total de citaciones

Fuente: Elaboración propia

Los resultados previos muestran que las investigaciones se publican casi exclusivamente en revistas asociadas al área de los negocios, la economía y la educación; se encuentra una participación menor de publicaciones ligadas a la psicología, y las ciencias y la tecnología. De este modo, el fenómeno se encuentra restringido al estudio de los campos de las ciencias sociales y las humanidades, lo que influye en el paradigma de investigación, junto a su abordaje ontológico, epistemológico y metodológico.

\section{Publicación de artículos por año}

La Figura 2 muestra el número de artículos publicados desde el año 2010 a 2020. Se puede observar que la investigación del efecto de la EE sobre la IE en estudiantes de nivel universitario, aun cuando muestra cierta oscilación, presenta un patrón creciente a lo largo de los años. El número de artículos publicados fue mínimo durante el periodo comprendido entre 2010 y 2014. Luego, hubo un aumento importante desde el año 2015 a 2019, en que el número de artículos pasó de 3 a 19 (533\%), con un promedio anual de 13 artículos. Esto demuestra el interés gradual de los investigadores e investigadoras por este campo de estudio. 


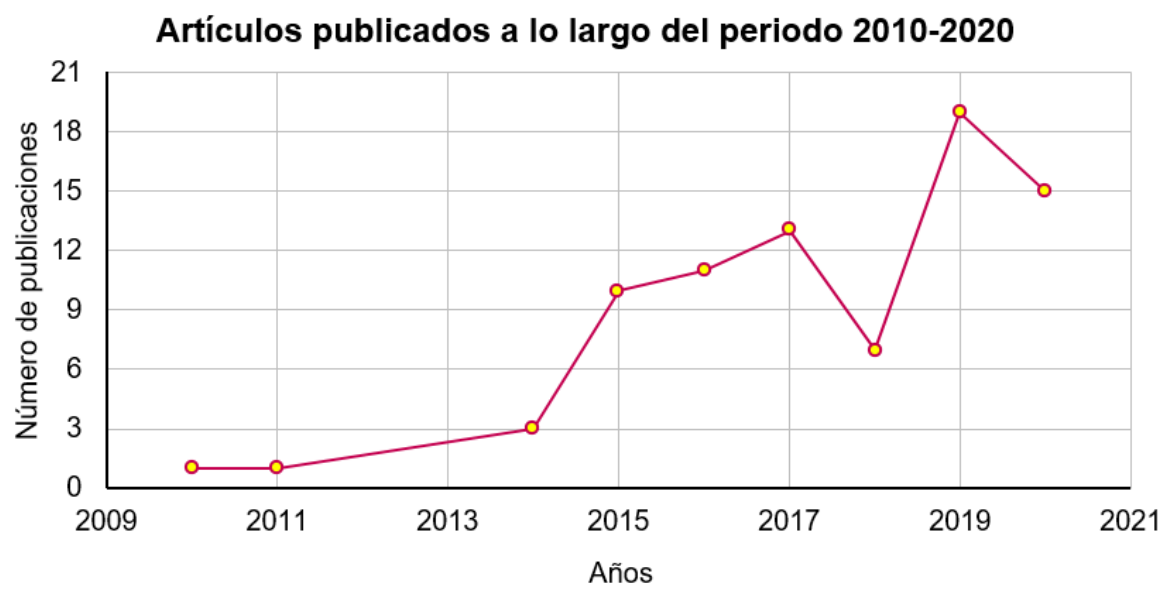

FIGURA 2

Artículos publicados a lo largo del periodo 2010-2020

Fuente: Elaboración propia

\section{Artículos más citados}

Dentro de los artículos con más de 80 citaciones destaca, en primer lugar (con 138 citas), la obra de Piperopoulos y Dimov (2015) denominada: Burst Bubbles or Build Steam? Entrepreneurship Education, Entrepreneurial Self-Efficacy, and Entrepreneurial Intentions. Esta investigación analizó la relación entre las creencias de autoeficacia percibida y la IE de 114 estudiantes de diferentes cursos de emprendimiento de una universidad británica. Por medio de la teoría del enfoque regulatorio, postuló que la naturaleza del curso (teórico o práctico) crea un marco motivacional distinto para el emprendimiento. Los resultados mostraron que una mayor autoeficacia se asocia con menores intenciones empresariales en los cursos orientados teóricamente y mayores intenciones empresariales en los cursos orientados a la práctica. Las personas autoras mencionan que si el objetivo es aumentar la cantidad de estudiantes que emprenden, entonces los programas de emprendimiento deben diseñarse e impartirse teniendo en cuenta el contexto, el contenido orientado a la práctica y la pedagogía de la enseñanza.

El segundo artículo más citado pertenece a Sánchez (2011), también con 138 citas. Este estudio evaluó el efecto de los programas de EE sobre las competencias emprendedoras y la IE de 863 discentes de Castilla y León en España ( 403 pertenecientes a un grupo experimental y 460 al grupo control). Los resultados mostraron que las personas que participaron del programa universitario aumentaron sus competencias y su intención de trabajar por cuenta propia, mientras que quienes participaron del grupo de control no lo hicieron. El autor resalta la importancia de la formación en las competencias consideradas clave para emprender y no solo la formación en los conocimientos y recursos necesarios para iniciar un negocio. Postula, además, que la formación en competencias empresariales implica, tácitamente, un componente inspirador, que impulsa la actitud y la intención por emprender.

El tercer artículo más citado es de Zhang, Duysters y Cloodt (2014), el cual tiene 115 citas. Este estudio, por medio de la Teoría del Comportamiento Planificado de Ajzen y el modelo de eventos empresariales de Shapero, así como la Teoría de la Cognición Empresarial, demostró que la EE tiene un impacto significativo y directo sobre la IE. También, destaca que el profesorado tiene un rol determinante en el fortalecimiento de la educación emprendedora, la cual, impartida adecuadamente, estimula la IE y mejora la capacidad de los potenciales emprendedores y emprendedoras para gestionar y desarrollar nuevas empresas.

Finalmente, el cuarto artículo más citado es de Karimi, Biemans, Lans, Chizari y Mulder (2016), que tiene 88 citas. El estudio, basado en la Teoría del Comportamiento Planificado de Ajzen, utilizó una encuesta 
ex ante y ex post para evaluar los impactos de los programas de educación emprendedora obligatorios y electivos sobre la intención de emprender. Los datos se recopilaron mediante un cuestionario aplicado a una muestra de 205 participantes de universidades iraníes. Si bien, ambos tipos de programas tuvieron impactos positivos sobre la IE, los resultados revelaron que solo los programas electivos aumentaron significativamente la IE. El estudio concluye que los tipos programas de educación emprendedora generan diferentes efectos dependiendo del contexto y los valores culturales del estudiantado.

La Tabla 3 presenta el listado con los diez artículos más citados para periodo de estudio.

TABLA 3

Artículos más citados sobre educación e intención emprendedora

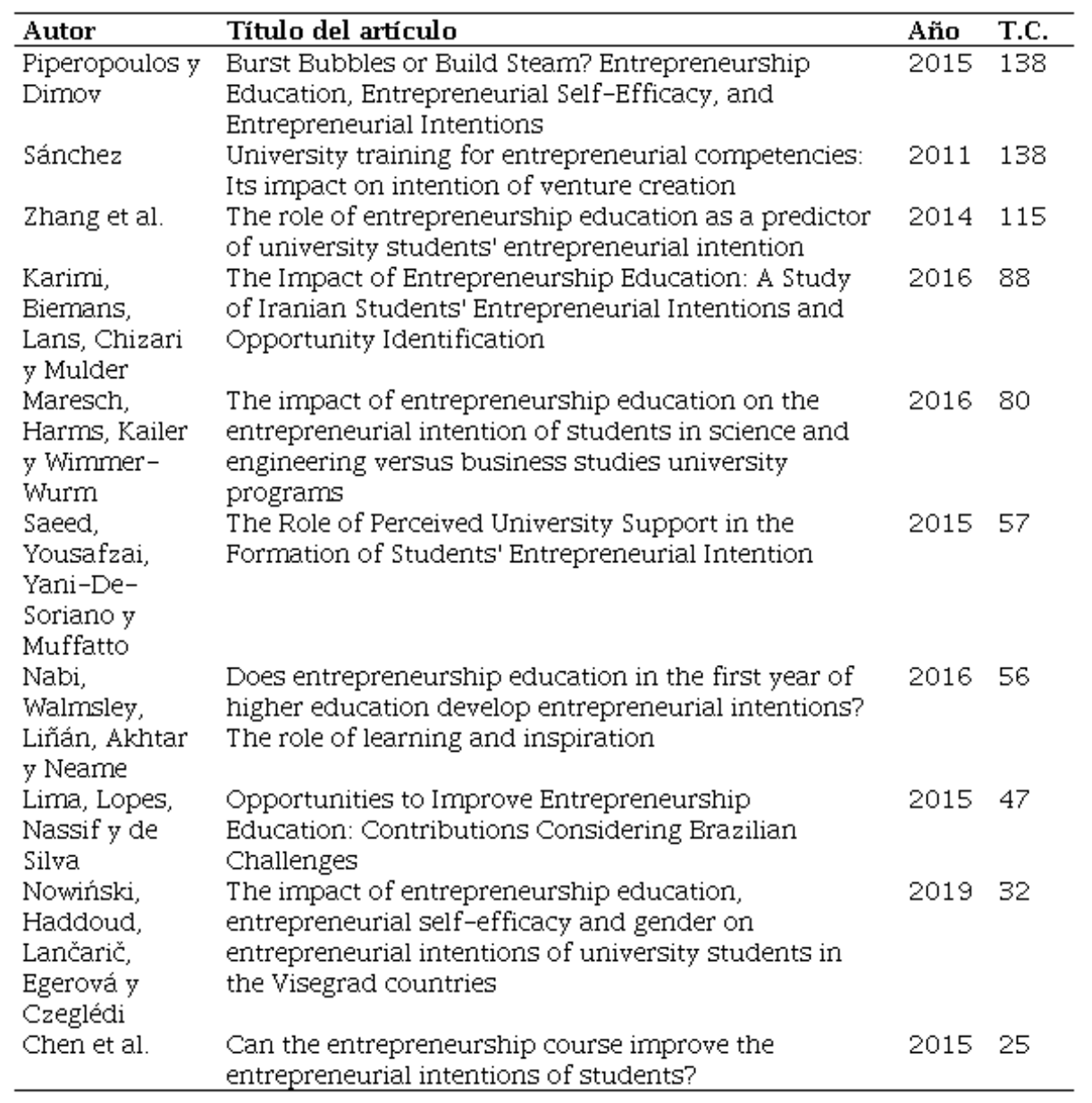

Fuente: Elaboración propia

Nota: T.C. $=$ Total de citaciones

\section{Países más prolíficos}

Todos los artículos de este estudio corresponden a 36 países diferentes. La Figura 3 muestra los diez países con mayor número de artículos sobre la influencia de la EE sobre la IE. China encabeza la lista con ocho artículos en total. Rumania, España, Pakistán, Malasia, Sudáfrica, Italia e India son los siguientes países con el máximo de trabajos. 


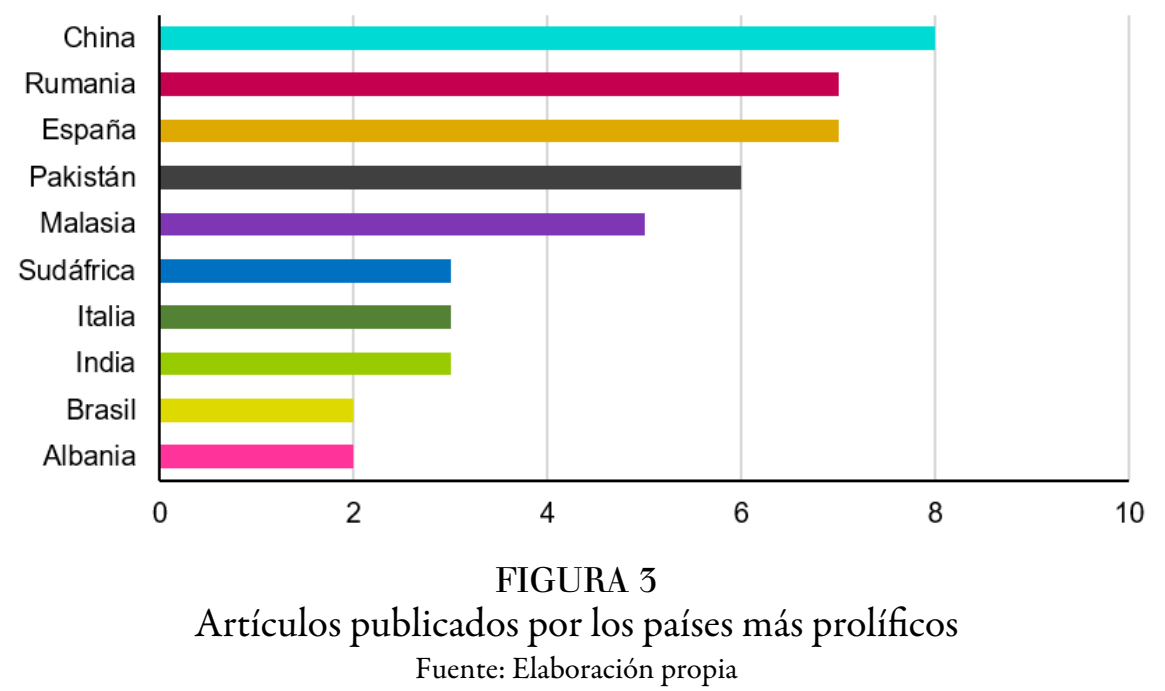

Fuente: Elaboración propia

La Figura 4 destaca los diversos países con sus respectivas contribuciones. Del total de artículos del estudio, más del 80\% (66) son aportados por personas autoras del continente asiático (33) y europeo (33), y un porcentaje menor es generado por autores del continente americano (6\%). Esta concentración de la producción científica en países de Asia y de Europa, abre la oportunidad para desarrollar estudios que analicen la influencia de la EE sobre la IE en otras zonas geográficas, que contrasten los hallazgos detectados con base a las diferencias culturales y de valores de sus habitantes.

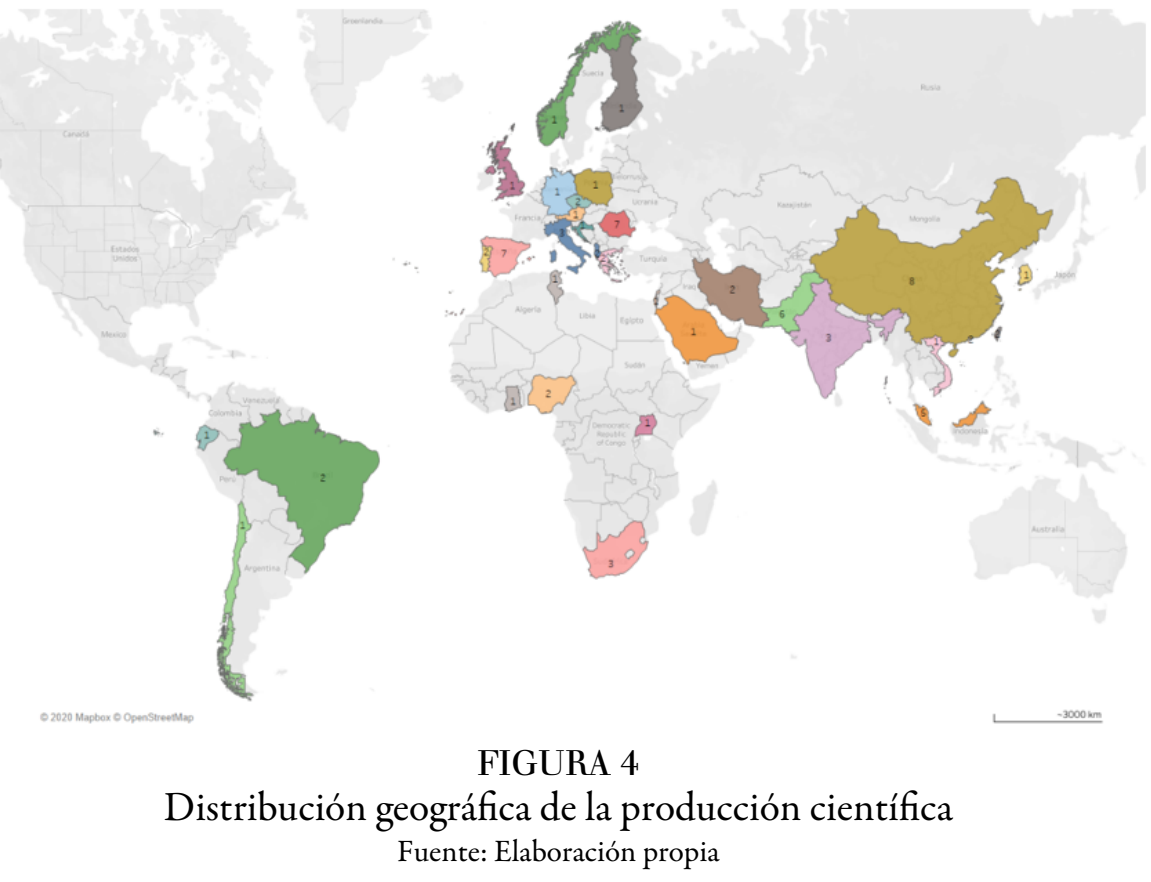

\section{Análisis de redes de coocurrencia de palabras clave}

Para determinar la relación de los términos claves de los artículos, se examinaron las coocurrencias en las publicaciones (van Eck et al., 2010). Para ello se utilizó el análisis de la red de coocurrencia, que permitió identificar los términos que han sido utilizados con mayor frecuencia en los diversos artículos referidos a la influencia de la EE sobre la IE. La rutina de minería de texto utilizada por VOSviewer construye una gráfica, 
donde la distancia entre diferentes términos representa la cercanía de la relación entre las diversas palabras clave (Laudano, Marzi y Caputo, 2018). Es decir, cuanto menor sea la distancia entre dos o más términos, más fuerte es su vinculación.

Para crear el mapa de red, se identificaron 54 términos que aparecieron más de tres veces en el conjunto de datos. El mapa muestra las diferentes palabras clave conectadas entre sí con varias líneas, las que representan que estas han coexistido en varios artículos del conjunto de datos. Véase la Figura 5.

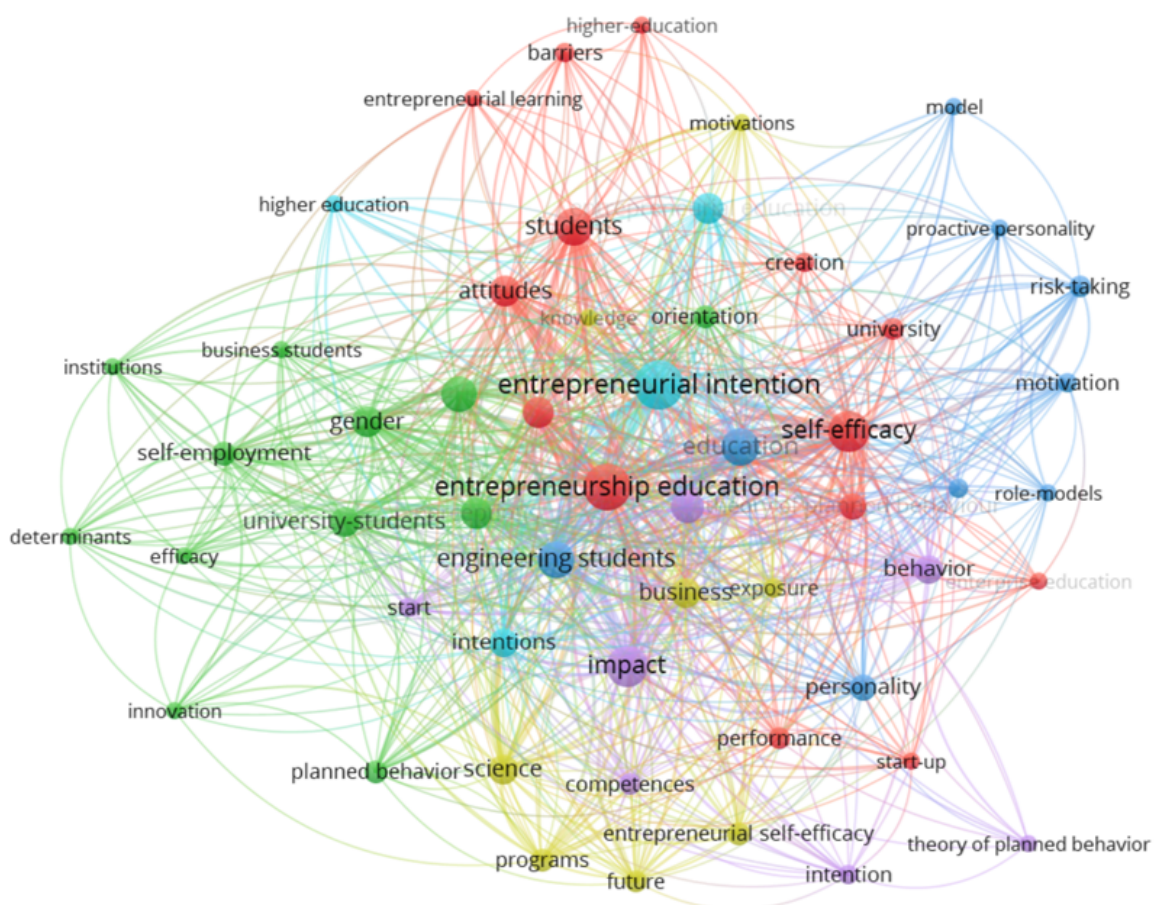

FIGURA 5

Mapa de red de coocurrencias de palabras clave Fuente: Elaboración propia

El examen del mapa de red encontró que casi todas las demás palabras clave que aparecen destacadas en bibliometría, véase la Tabla 4, han coexistido con los términos y sinónimos de entrepreneurship education y entrepreneurial intention. Las palabras clave que han aparecido la mayor cantidad de veces sugieren que se han realizado más investigaciones en tales áreas. 
TABLA 4

Palabras clave más frecuentes y sus vínculos totales

\begin{tabular}{|c|c|c|c|c|c|}
\hline Palabra clave & $\mathrm{Oa}$ & Vo & Palabra clave & $\mathrm{Oa}$ & Vo \\
\hline $\begin{array}{l}\text { 1. entrepreneurial } \\
\text { intention }\end{array}$ & 42 & 250 & 11. attitudes & 13 & 116 \\
\hline $\begin{array}{l}\text { 2. entrepreneurship } \\
\text { education }\end{array}$ & 37 & 231 & 12. gender & 13 & 112 \\
\hline 3. impact & 27 & 210 & 13. models & 13 & 94 \\
\hline 4. self-efficacy & 27 & 183 & 14. university-students & 12 & 96 \\
\hline 5. education & 22 & 145 & 15. business & 12 & 95 \\
\hline 6. Students & 21 & 124 & 16. science & 12 & 86 \\
\hline 7. engineering students & 19 & 143 & 17. behavior & 10 & 77 \\
\hline $\begin{array}{l}\text { 8. entrepreneurial } \\
\text { intentions }\end{array}$ & 18 & 89 & 18. intentions & 10 & 62 \\
\hline 9. entrepreneurship & 16 & 105 & $\begin{array}{l}\text { 19. theory of planned } \\
\text { behavior }\end{array}$ & 8 & 76 \\
\hline $\begin{array}{l}\text { 10. entrepreneurial } \\
\text { education }\end{array}$ & 13 & 59 & 20. personality & 8 & 54 \\
\hline
\end{tabular}

Fuente: Elaboración propia

Nota $\mathrm{Oa}=$ Ocurrencia; $\mathrm{Vo}=$ Vínculos

La Figura 6 muestra las diversas palabras clave que ocurren como máximo y que han coexistido con el concepto entrepreneurship education. Este mapa puede proporcionar una breve visión sobre las diversas temáticas y áreas; los investigadores e investigadoras han centrado su atención con mayor frecuencia en relación con el estudio de la EE y la IE. Por ejemplo, la coocurrencia de las palabras clave engineeringstudents o business students y entrepreneurship education sugiere que se han realizado varias investigaciones sobre educación emprendedora en estudiantes de las áreas de ingeniería y de los negocios. De manera similar, la coexistencia de las palabras clave como theory of planned behavior o planned behavior y entrepreneurship education sugiere que los estudios han utilizado como marco teórico, para evaluar la influencia de la educación emprendedora, la Teoría del Comportamiento Planificado (TPB, por sus siglas en inglés, Theory of Planned Behavior). También, vinculadas a los términos entrepreneurship education y entrepreneurial intention coexisten diversos factores psicosociales considerados determinantes de la EE y la IE, tales como la autoeficacia (self-efficacy), la personalidad (personality) y la actitud (attitude). 


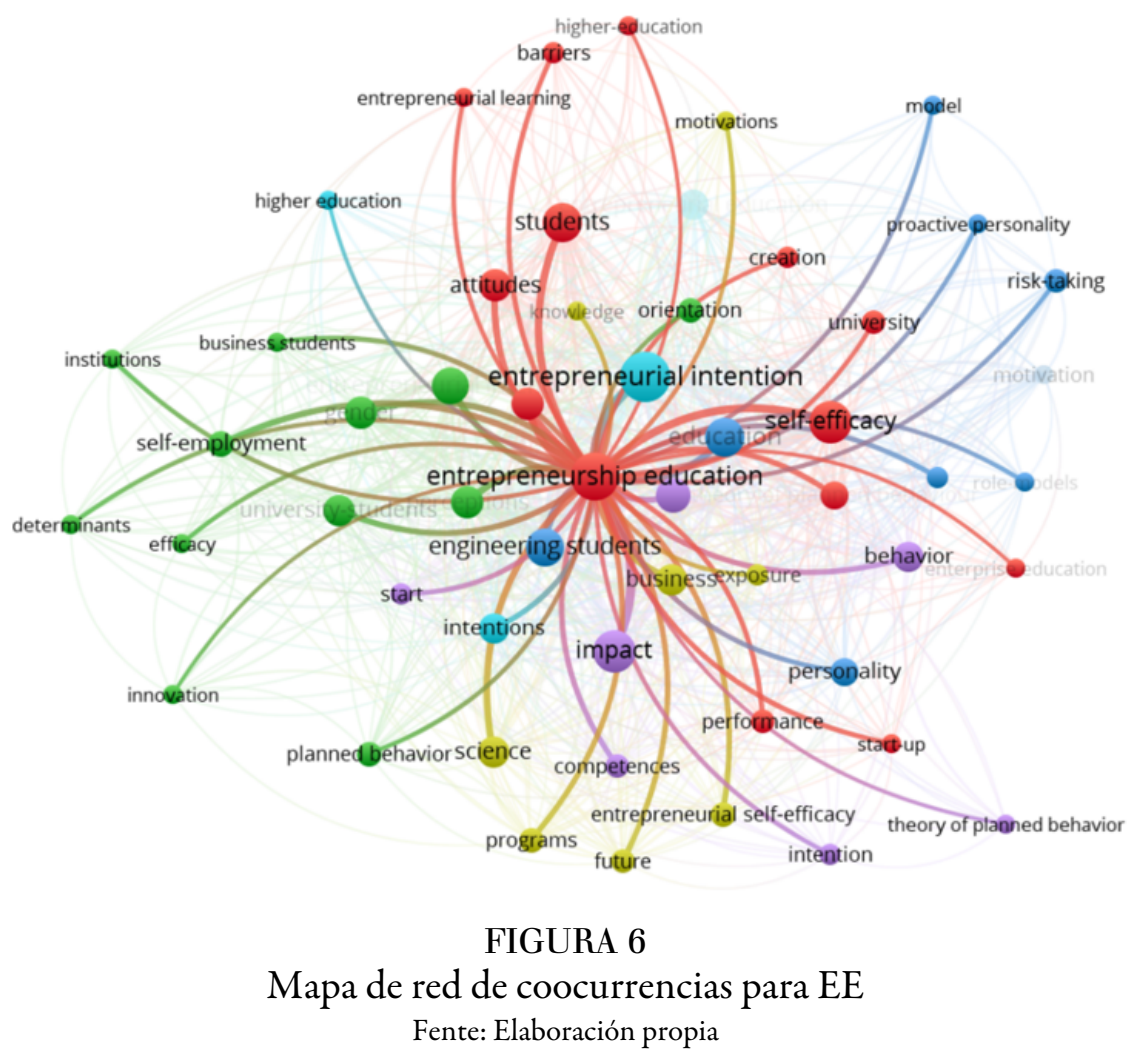

\section{Opción teórica, metodología y hallazgos}

En primer lugar, según su opción teórica, se observó que la mayoría de los trabajos utilizan como marco referencial el modelo de Comportamiento Planificado de Ajzen (1991) (Galvão, Marques y Ferreira, 2020; Malebana, 2016; Maresch Harms, Kailer y Wimmer-Wurm, 2016; Paray y Kumar, 2020; Passaro, Quinto y Thomas, 2018; Sun, Lo, Liang y Wong, 2017; Zovko, Dulčić y Bilić, 2020), por sobre otros modelos clásicos que explican la intención emprendedora (Krueger y Brazeal, 1994; Shapero y Sokol, 1982). Al respecto, cabe destacar que la Teoría del Comportamiento Planificado de Ajzen (1991) tiene su origen en los postulados de la Teoría Cognitiva Social de Bandura (Bandura, 1977), especialmente en aquellos referidos a las creencias de autoeficacia; no obstante, la TPB plantea la influencia de tres variables psicosociales que afectan la intención las cuales son: actitud, norma subjetiva y control del comportamiento percibido. Es, precisamente, el valor explicativo del modelo causal de Ajzen (1991) y la cuantía de evidencia empírica que ha logrado desarrollarse en torno a ella, lo que le ha permitido posicionarse en el ámbito de la predicción del comportamiento.

De la aplicación del modelo, los resultados asociados a los tipos de creencias que explican su teoría (actitud, norma subjetiva y control conductual percibido), resalta el rol de la educación emprendedora para contribuir a la formación de las actitudes, lo que incrementaría la intención por emprender (Paray y Kumar, 2020; Sun et al., 2017; Zovko et al., 2020).

En segundo lugar, el enfoque metodológico fue esencialmente cuantitativo (Cera, Mlouk, Cera y Shumeli, 2020; Longva, Strand y Pasquine, 2020; Jena, 2020; Haddoud, Onjewu, Nowinski y Alammari, 2020; SoriaBarreto et al., 2016; Badri y Hachicha, 2019; Paray y Kumar, 2020). Se registraron pocos estudios que abordaran el fenómeno desde una perspectivas cualitativa o mixta (Jamaluddin, Ali, Abdul Kadir y Kamis, 2019; Nabi, Walmsley, Liñán, Akhtar y Neame, 2016; Egerová, Eger y Mičík, 2017; Hamzah, Yahya, Sarip y Adnan, 2016; Fang y Chen, 2019). En este sentido, destacaron como técnicas de análisis de datos los modelos de ecuaciones estructurales - SEM (Karimi et al., 2016; Kakouris, 2016; Sun et al., 2017; Passaro 
et al., 2018; Galvão et al., 2020; Nowiński et al., 2019; Jena, 2020), las regresiones logit/probit (Badri y Hachicha, 2019; Maresch et al., 2016; Vodă y Florea, 2019; Vélez, Bustamante, Loor y Afcha, 2020; Zhang et al., 2014; Piperopoulos y Dimov, 2015) y ANOVAS (Popescu, Bostan, Robu, Maxim y Diaconu, 2016; Soria-Barreto et al., 2016; Soomro, Memon y Bukhari, 2020; Lima, Lopes, Nassif y de Silva, 2015), cuya información se recolectó partir de cuestionarios aplicados a estudiantes, y en menor proporción utilizaron muestras combinadas con discentes de postgrado (Kakouris 2016; Piperopoulos y Dimov, 2015; Lima et al., 2015) o docentes (Passaro et al., 2018). También se pudo constatar que la mayoría de las investigaciones fueron de corte transversal, siendo escasos los ejemplos relacionados a investigaciones de tipo longitudinal (Soria-Barreto et al., 2016; Varamäki, Joensuu, Tornikoski y Viljamaa, 2015).

En relación a los resultados, se observa que los efectos de la EE sobre la IE son diversos (Piperopoulos y Dimov, 2015) y, por tanto, no concluyentes. No obstante, fue posible advertir una tendencia mayor a destacar la influencia significativa y positiva de la EE, tanto de directa como indirectamente, y, en menor medida, una influencia desfavorable o insignificante. La Tabla 5 agrupa a los referentes más citados según el tipo de hallazgo sobre la influencia de la enseñanza de emprendimiento: favorable (directa o indirectamente), desfavorable (negativa o no significativa) o variable.

TABLA 5

Referentes más citados según el tipo influencia de la EE sobre la IE

\begin{tabular}{|c|c|c|c|}
\hline Influencia & Autor & Revista* & T.C. \\
\hline \multirow[t]{11}{*}{ Gr.1. Favorable } & Sánchez (2011) & IEMJ & 138 \\
\hline & Zhang et al. (2014) & IEMJ & 115 \\
\hline & Karimi et al. (2016) & JSBM & 88 \\
\hline & Maresch et al. (2016) & TFSC & 80 \\
\hline & Saeed et al. (2015) & JSBM & 57 \\
\hline & Nowiński et al. (2019) & SHE & 32 \\
\hline & Passaro et al. (2018) & $\mathrm{JIC}$ & 24 \\
\hline & Yemini y Haddad (2010) & IJEE & 24 \\
\hline & $\begin{array}{l}\text { Iglesias-Sánchez, Jambrino- } \\
\text { Maldonado, Velasco y Kokash (2016) }\end{array}$ & $\mathrm{E}+\mathrm{T}$ & 21 \\
\hline & Popescu et al. (2016) & Sus & 17 \\
\hline & Teixeira y Forte (2017) & RMS & 10 \\
\hline \multirow[t]{10}{*}{ Gr.2. Desfavorable } & Lima et al. (2015) & JSBM & 47 \\
\hline & Chen et al. (2015) & IEMJ & 25 \\
\hline & Soria-Barreto et al. (2016) & FU & 17 \\
\hline & Varamäki et al. (2015) & JSBED & 14 \\
\hline & Galvão, Marques y Marques (2018) & $\mathrm{E}+\mathrm{T}$ & 8 \\
\hline & $\begin{array}{l}\text { Camacho-Miñano y del Campo } \\
\text { (2015) }\end{array}$ & $E+T$ & 8 \\
\hline & Vodă y Florea (2019) & Sus & 7 \\
\hline & Sitaridis y KitSios (2017) & JIBED & 5 \\
\hline & Fang y Chen (2019) & Sus & 3 \\
\hline & Hamzah et al. (2016) & PRPRJ & 3 \\
\hline \multirow[t]{2}{*}{ Gr. 3. Variable } & Piperopoulos y Dimov (2015) & JSBM & 138 \\
\hline & Nabi et al. (2016) & SHE & 56 \\
\hline
\end{tabular}

Fuente: Elaboración propia

Nota:* IEMJ: International Entrepreneurship and Management Journal, JSBM: Journal of Small Business Management, TFSC: Technological Forecasting and Social Change, JSBM: Journal of Small Business Management, SHE: Studies in Higher Education, JIC: Journal of Intellectual Capital, IJEE: International Journal of Engineering

Education, E+T: Education and Training, Sus: Sustainability, RUKSJ: Rusc-Universities and Knowledge Society Journal, ES: Educational Studies, RMS: Review of Managerial Science, FU: Formación Universitaria, JSBED: Journal of Small Business and Enterprise Development, JIBED: Journal for International Business and Entrepreneurship Development, PRPRJ: Pacific Rim Property Research Journal ; T.C. $=$ Total de citaciones

Por último, la Figura 7 resume el efecto agregado de la EE sobre la IE de acuerdo al tipo de contribución detectada en las diversas publicaciones estudiadas (contribución positiva o no). 


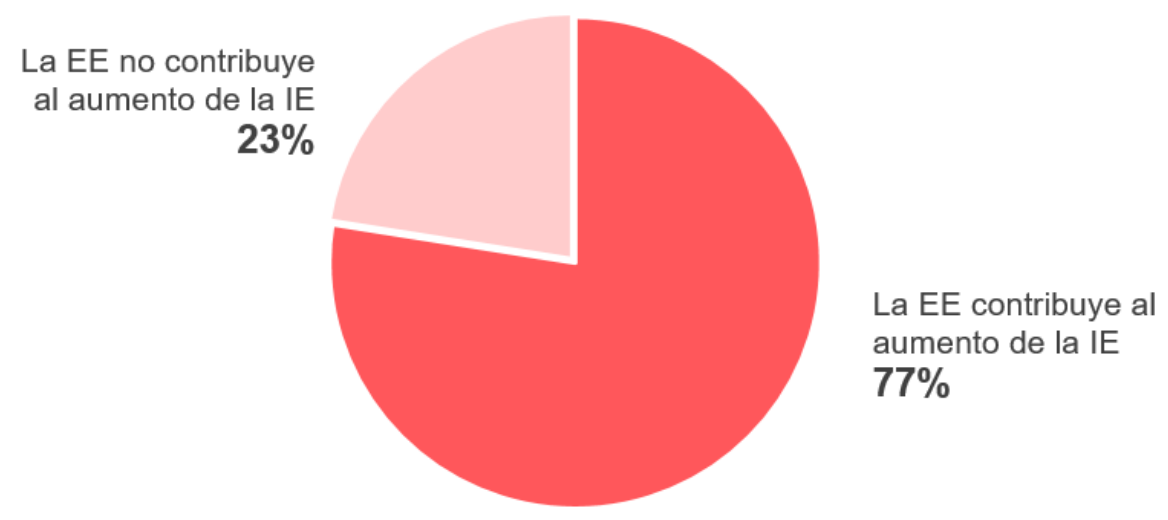

FIGURA 7

Contribución de la educación sobre la intención emprendedora

Fuente: Elaboración propia

En la gráfica se advierte que, de los 80 artículos revisados, el $77 \%$ señala que la EE contribuye a aumentar, directa o indirectamente, la IE del estudiantado. Mientras que el $23 \%$ restante, resulta en un aporte negativo o no significativo. Así, los resultados permiten evidenciar la influencia positiva de la EE sobre la IE, lo que la posiciona como una variable explicativa relevante que ayudaría a comprender por qué algunas personas emprenden y otras no.

\section{Conclusiones}

Con base en el análisis de los resultados se concluye, primero, que los estudios encuentran, principalmente, una influencia significativa y positiva de la enseñanza de emprendimiento sobre la intención emprendedora. No obstante, los resultados dependen de aspectos contextuales y del tipo de enseñanza. Dentro de las causas que explican la variabilidad de los resultados, cabe mencionar principalmente factores pedagógicos, como el tipo de clase (teórica o práctica), el contenido (centrado o no en competencias), el foco de enseñanza (orientado o no a la acción) y la modalidad del programa (obligatorio o no). En general, se aprecia que la educación emprendedora resulta más efectiva cuando posee un enfoque práctico, orientado a la acción y el desarrollo de competencias emprendedoras (Musetsho y Lethoko, 2017; Olokundun et al., 2018; Piperopoulos y Dimov, 2015; Sánchez, 2011). También, cabe destacar que existen aspectos psicosociales, demográficos y culturales del alumnado, que influyen de manera directa e indirecta sobre la IE y el efecto de la EE. Entre estos factores resaltan la actitud hacia el emprendimiento, la autoeficacia, la personalidad proactiva, la necesidad de logro y los valores culturales. Además de factores demográficos, tales como el género, la presencia de modelos de referencia (amistades y familiares), la experiencia previa en emprendimiento y el pasado laboral (haber trabajado o no). En tal perspectiva, el problema de efectividad de los programas de emprendimiento residiría en la manera en que este es enseñado y, por lo tanto, plantea el desafío de responder cómo llevar a cabo una pedagogía emprendedora idónea, alineada al contexto y características del alumnado, y al ambiente dinámico presente en el mundo.

Por lo demás, como la mayoría de la producción científica proviene de Asia y Europa, existe la oportunidad de desarrollar estudios en otras zonas geográficas que contrasten los hallazgos detectados. Segundo, la metodología utilizada para analizar la influencia de la EE sobre la IE se centra en un paradigma positivista, de enfoque cuantitativo, lo que devela la posibilidad de abordar el fenómeno desde una metodología mixta o cualitativa. Las aproximaciones cualitativas podrían brindar un complemento apropiado para comprender más profundamente la contribución de la educación sobre las intenciones de las personas, esto de acuerdo con los aspectos psicográficos, tales como los valores, la personalidad y los estilos de vida. De este modo, un 
enfoque mixto permitiría avanzar hacia una comprensión más integral del fenómeno de la formación de la intención emprendedora y la conducta. Tercero, se podría incorporar también como fuente de información complementaria, al cuerpo docente y no solo al estudiantado. Esta visión participativa es necesaria para abordar la incidencia de las estrategias de enseñanza en la motivación del alumnado y sus procesos de aprendizaje. Y, cuarto, gran parte de las investigaciones poseen un alcance transversal, por lo que podría examinarse la evolución del efecto de la enseñanza del emprendimiento mediante el desarrollo de estudios longitudinales. Ello permitiría analizar los efectos de largo plazo de la educación emprendedora sobre la intención y posterior acción empresarial.

\section{RECOMENDACIONES}

Con relación a los hallazgos del estudio, sería recomendable que futuras investigaciones se enfoquen en la revisión de los factores determinantes de la efectividad de la educación emprendedora. En especial del escrutinio de los aspectos pedagógicos que influyen en la actitud del estudiantado hacia el emprendimiento. Centrar los esfuerzos en examinar el tipo de educación emprendedora impartida, las metodologías de enseñanza usadas por el profesorado, los sistemas evaluativos, la didáctica, el uso de las tecnologías de la información y comunicación, entre otras. Pues si bien se pudo demostrar una tendencia favorable de la EE sobre la IE, no se observó un estudio más exhaustivo de aquellos aspectos que caracterizan a una educación emprendedora efectiva. Esto toma más relevancia si se considera el rol fundamental que juegan las organizaciones educativas en la gestión de los factores pedagógicos de la enseñanza.

De este, para que la EE tenga una influencia positiva y significativa sobre la IE, se requiere de una visión integral del proceso de enseñanza y aprendizaje, que configure un proyecto de educación emprendedora con participación activa del estudiantado, el profesorado y la institución educativa.

En cuanto a la metodología, se torna importante revisar otras bases de datos científicas, como Scopus o SciELO, lo que permitiría obtener un panorama geográfico más amplio, y con mayor presencia de autores de habla hispana.

Por último, cabe destacar que se observa un espacio relevante para abordar la influencia de la EE sobre la IE desde enfoques más diversos e integrales, que sumen a la aproximación cuantitativa, estudios de tipo cualitativo y mixto. Esto brindaría un mayor potencial para analizar, desde diferentes matices, los aspectos determinantes del impacto de la educación emprendedora.

\section{REFERENCIAS BIBLIOGRÁFICAS}

Ajzen, I. (1991). The theory of planned behavior. Organizational Behavior and Human Decision Processes, 50(2), 179211. doi: https://doi.org/10.1016/0749-5978(91)90020-T

Badri, R. y Hachicha, N. (2019). Entrepreneurship education and its impact on students' intention to start up: A sample case study of students from two Tunisian universities. The International Journal of Management Education, 17(2), 182-190. doi: https://doi.org/10.1016/j.ijme.2019.02.004

Bandura, A. (1977). Social Learning Theory. New Jersey: Prentice-Hall.

Camacho-Miñano, M. y del Campo, C. (2015). The role of creativity in entrepreneurship: an empirical study on business undergraduates. Education + Training, 59(7/8), 672-688. doi: https://doi.org/10.1108/ET-08-2016 $-0132$

Čapienè, A. y Ragauskaité, A. (2017). Entrepreneurship education at university: Innovative models and current trends. Research for Rural Development, 2, 284-291. doi: https://doi.org/10.22616/rrd.23.2017.080

Çera, G. y Çera, E. (2020). Intention to start a business and entrepreneurship education programme: a pre- and postprogramme research design.Journal of Enterprising Communities: People and Places in the GlobalEconomy, 14(4), 603-619. doi: https://doi.org/10.1108/JEC-05-2020-0095 
Çera, G., Mlouk, A., Çera, E. y Shumeli, A. (2020). The impact of entrepreneurship education on entrepreneurial intention. A quasi-experimental research design. Journal of Competitiveness, 12(1), 39-56. doi: https://doi.org /10.7441/joc.2020.01.03

Chen, C., Hsiao, C., Chang, C., Chou, M., Chen, P. y Shen, H. (2015). Can the entrepreneurship course improve the entrepreneurial intentions of students? International Entrepreneurship and Management Journal, 11 (3), 557569. doi: https://doi.org/10.1007/s11365-013-0293-0

da Silva Moreira, A., Loiola, E. y Guedes, M. (2017). Individual and contextual predictors of entrepreneurial intention among undergraduates: a literature review. Cadernos EBAPE.BR, 15(2), 292-308. doi: https://doi.org/10.159 0/1679-395159595

Egerová, D., Eger, L. y Mič́k, M. (2017). Does entrepreneurship education matter? Business students' perspectives. Tertiary Education and Management, 23(4), 319-333. doi: https://doi.org/10.1080/13583883.2017.1299205

Fang, C. y Chen, L. (2019). Exploring the entrepreneurial intentions of science and engineering students in China: A Q methodology study. Sustainability, 11(10). doi: https://doi.org/10.3390/su11102751

Galvão, A., Marques, C. y Ferreira, J. J. (2020). The role of entrepreneurship education and training programmes in advancing entrepreneurial skills and new ventures. European Journal of Training and Development, 44(6/7), 595-614. doi: https://doi.org/10.1108/EJTD-10-2019-0174

Galvão, A., Marques, S. y Marques, P. (2018). Antecedents of entrepreneurial intentions among students in vocational training programmes. Education and Training, 60(7/8), 719-734. doi: https://doi.org/10.1108/ET-03-20170034

Haddoud, M. Y., Onjewu, A. K. E., Nowinski, W. y Alammari, K. (2020). Assessing the role of entrepreneurship education in regulating emotions and fostering implementation intention: evidence from Nigerian universities. Studies in Higher Education, 1-19. doi: https://doi.org/10.1080/03075079.2020.1758652

Hamzah, H., Yahya, Z., Sarip, A. G. y Adnan, Y. M. (2016). Impact of entrepreneurship education programme (EEP) on entrepreneurial intention of real estate graduates. Pacific Rim Property Research Journal, 22(1), 17-29. doi: https://doi.org/10.1080/14445921.2016.1158897

Iglesias-Sánchez, P. P., Jambrino-Maldonado, C., Velasco, A. P. y Kokash, H. (2016). Impact of entrepreneurship programmes on university students. Education and Training, 58(2), 209-228. doi: https://doi.org/10.1108/E T-01-2015-0004

Jamaluddin, R., Ali, H., Abdul, S. y Kamis, A. (2019). Impact of fashion entrepreneurship programme on entrepreneurial interests, intention and competencies. Journal of Technical Education and Training, 11(1), 119128. doi: https://doi.org/10.30880/jtet.2019.11.01.015

Jena, K. (2020). Measuring the impact of business management Student's attitude towards entrepreneurship education on entrepreneurial intention: A case study. Computers in Human Behavior, 107. doi: https://doi.org/10.1016 /j.chb.2020.106275

Kakouris, A. (2016). Exploring entrepreneurial conceptions, beliefs and intentions of Greek graduates. International Journal of Entrepreneurial Behavior \& Research, 22(1), 109-132. doi: https://doi.org/10.1108/IJEBR-07-201 4-0137

Karimi, S., Biemans, A., Lans, T., Chizari, M. y Mulder, M. (2016). The impact of entrepreneurship education: A study of iranian students' entrepreneurial intentions and opportunity identification. Journal of Small Business Management, 54(1), 187-209. doi: https://doi.org/10.1111/jsbm.12137

Krueger, F. y Brazeal, V. (1994). Entrepreneurial Potential and Potential Entrepreneurs. Entrepreneurship Theory and Practice, 91-104. Recuperado de https://bit.ly/3dEYYGF

Lanero, A., Vázquez, L., Gutiérrez, P. y García, P. (2011). Evaluación de la conducta emprendedora en estudiantes universitarios: implicaciones para el diseño de programas académicos. Pecunia, (12), 219-243. doi: https://doi .org/10.18002/pec.v0i12.623

Laudano, M. C., Marzi, G. y Caputo, A. (2018). A decade of the International Journal of Entrepreneurship and Small Business: a bibliometric analysis. International Journal of Entrepreneurship and Small Business, 33(2), 289-314. doi: https://doi.org/10.1504/ijesb.2018.10010981 
Lima, E., Lopes, M., Nassif, V. y de Silva, D. (2015). Opportunities to improve entrepreneurship education: Contributions considering brazilian challenges. Journal of Small Business Management, 53(4), 1033-1051. doi: https://doi.org/10.1111/jsbm.12110

Longva, K. K., Strand, Ø. y Pasquine, M. (2020). Entrepreneurship education as an arena for career reflection: the shift of students' career preferences after a business planning course. Education and Training, 62(7/8), 877-896. doi: https://doi.org/10.1108/ET-08-2019-0187

Malebana, M. J. (2016). Does entrepreneurship education matter for the enhancement of entrepreneurial intention? Southern African Business Review, 20(1), 365-387. doi: https://doi.org/10.25159/1998-8125/6056

Maresch, D., Harms, R., Kailer, N. y Wimmer-Wurm, B. (2016). The impact of entrepreneurship education on the entrepreneurial intention of students in science and engineering versus business studies university programs. Technological Forecasting and Social Change, 104, 172-179. doi: https://doi.org/10.1016/j.techfore.2015.11.0 06

Montilla, L. (2012). Análisis bibliométrico sobre la producción científica archivística en la Red de Revistas Científicas de América Latina y el Caribe (Redalyc) durante el período 2001-2011. Biblios: Journal of Librarianship and Information Science, (48), 1-11. doi: https://doi.org/10.5195/biblios.2012.65

Musetsho, T. y Lethoko, M. (2017). An Evaluative Study on the Effect of Entrepreneurial Education Curriculum on Students at the University of Venda, South Africa. Independent Journal of Teaching and Learning, 12(1), 7489. Recuperado de https://bit.ly/33aHigs

Nabi, G., Walmsley, A., Liñán, F., Akhtar, I. y Neame, C. (2016). Does entrepreneurship education in the first year of higher education develop entrepreneurial intentions? The role of learning and inspiration. Studies in Higher Education, 43(3), 452-467. doi: https://doi.org/10.1080/03075079.2016.1177716

Norris, M. y Oppenheim, C. (2007). Comparing alternatives to the Web of Science for coverage of the social sciences' literature. Journal of Informetrics, 1(2), 161-169. doi: https://doi.org/10.1016/j.joi.2006.12.001

Nowiński, W., Haddoud, M. Y., Lančarič, D., Egerová, D. y Czeglédi, C. (2019). The impact of entrepreneurship education, entrepreneurial self-efficacy and gender on entrepreneurial intentions of university students in the Visegrad countries. Studies in Higher Education, 44(2), 361-379. doi: https://doi.org/10.1080/03075079.201 7.1365359

Olokundun, M., Moses, C., Iyiola, O., Ibidunni, S., Ogbari, M., Peter, F. y Borishade, T. (2018). The effect of non traditional teaching methods in entrepreneurship education on students entrepreneurial interest and business startups: A data article. Data in Brief, 19, 16-20. doi: https://doi.org/10.1016/j.dib.2018.04.142

Paray, Z. A. y Kumar, S. (2020). Does entrepreneurship education influence entrepreneurial intention among students in HEI's?: The role of age, gender and degree background. Journal of International Education in Business, 13(1), 55-72. doi: https://doi.org/10.1108/JIEB-02-2019-0009

Passaro, R., Quinto, I. y Thomas, A. (2018). The impact of higher education on entrepreneurial intention and human capital.Journal of Intellectual Capital, 19(1), 135-156. doi: https://doi.org/10.1108/JIC-04-2017-0056

Piperopoulos, P. y Dimov, D. (2015). Burst Bubbles or Build Steam? Entrepreneurship Education, Entrepreneurial Self-Efficacy, and Entrepreneurial Intentions. Journal of Small Business Management, 53(4), 970-985. doi: htt ps://doi.org/10.1111/jsbm.12116

Popescu, C. C., Bostan, I., Robu, I.-B., Maxim, A. y Diaconu, L. (2016). An analysis of the determinants of entrepreneurial intentions among students: A Romanian case study. Sustainability, 8(8), 1-22. doi: https://d oi.org/10.3390/su8080771

Saeed, S., Yousafzai, S. Y., Yani-De-Soriano, M. y Muffatto, M. (2015). The Role of Perceived University Support in the Formation of Students' Entrepreneurial Intention. Journal of Small Business Management, 53(4), 1127 1145. doi: https://doi.org/10.1111/jsbm.12090

Sánchez, J. C. (2011). University training for entrepreneurial competencies: Its impact on intention of venture creation. International Entrepreneurship and Management Journal, 7, 239-254. doi: https://doi.org/10.1007/ s11365-010-0156-x 
Shapero, A. y Sokol, L. (1982). The Social Dimensions of Entrepreneurship. Encyclopedia of Entrepreneurship, (1982), 72-90. doi: https://bit.ly/3aLWgxx

Sitaridis, I. y Kitsios, F. (2017). Entrepreneurial intentions of information technology students: the theory of planned behaviour, the role of gender and education. Journal for International Business and Entrepreneurship Development, 10(3), 3-16. doi: https://doi.org/10.1504/jibed.2017.085508

Soomro, M. A., Memon, M. S. y Bukhari, N. S. (2019). Entrepreneurial education and its impact on entrepreneurial intentions: A comparative analysis of business graduates of public and private universities of Sindh. Pacific Business Review International, 11 (6), 35-46. Recuperado de https://bit.ly/3jNHng4

Soria-Barreto, K., Zuniga-Jara, S. y Ruiz-Campo, S. (2016). Educación e intención emprendedora en estudiantes universitarios: Un caso de estudio. Formación Universitaria, 9(1), 25-34. doi: https://doi.org/10.4067/S0718 $-50062016000100004$

Stamboulis, Y. y Barlas, A. (2014). Entrepreneurship education impact on student attitudes. International Journal of Management Education, 12(3), 365-373. doi: https://doi.org/10.1016/j.ijme.2014.07.001

Sun, H., Lo, T., Liang, B. y Wong, B. (2017). The impact of Entrepreneurial Education on Entrepreneurial Intention of Engineering Students in Hong Kong. Management Decision, 55(7), 1371-1393. doi: https://doi.org/10.11 08/MD-06-2016-0392

Teixeira, C. y Forte, P. (2017). Prior education and entrepreneurial intentions: the differential impact of a wide range of fields of study. Review of Managerial Science, 11, 353-394. doi: https://doi.org/10.1007/s11846-015-0188-2

van Eck, N. J., Waltman, L., Dekker, R. y van den Berg, J. (2010). A Comparison of TwoTechniques for Bibliometric Mapping: Multidimensional Scaling and VOS. Journal of the American Society for Information Science and Technology, 61(12), 2405-2416. doi: https://doi.org/10.1002/asi.21421

Varamäki, E., Joensuu, S., Tornikoski, E. y Viljamaa, A. (2015). The development of entrepreneurial potential among higher education students. Journal of Small Business and Enterprise Development, 22(3), 563-589. doi: https:/ /doi.org/10.1108/JSBED-02-2012-0027

Vélez, I., Bustamante, A., Loor, A. y Afcha, M. (2020). La educación para el emprendimiento como predictor de una intención emprendedora de estudiantes universitarios. Formación universitaria, 13(2), 63-72. doi: https://doi .org/10.4067/s0718-50062020000200063

Vodă, I. y Florea, N. (2019). Impact of personality traits and entrepreneurship education on entrepreneurial intentions of business and engineering students. Sustainability, 11(4). doi: https://doi.org/10.3390/SU11041192

Yemini, M. y Haddad, J. (2010). Engineer-entrepreneur: Combining technical knowledge with entrepreneurship education-The Israeli case study. International Journal of Engineering Education, 26(5), 1220-1229. Recuperado de https://bit.ly/3idy6NK

Zhang, Y., Duysters, G. y Cloodt, M. (2014). The role of entrepreneurship education as a predictor of university students' entrepreneurial intention. International Entrepreneurship and Management Journal, 10(3), 623-641. doi: https://doi.org/10.1007/s11365-012-0246-z

Zovko, L., Dulčić, Ž. y Bilić, I. (2020). Determinants of students’ entrepreneurial intention: An empirical research. Journal of Contemporary Management Issues, 25(1), 25-44. doi: https://doi.org/10.30924/mjcmi.25.1.2

\section{INFORMACIÓN ADICIONAL}

Cómo citar: Araya-Pizarro, S.C. (2021). Influencia de la educación emprendedora sobre la intención de emprender del alumnado universitario. Revista Educación, 45(2). Recuperado de http://doi.org/10.15517 /revedu.v45i1.43748 